\title{
Abordar la violencia sexual basada en el género (SGBV) contra niñas adolescentes
}

Sajeda Amin

Population Council

Althea D. Anderson

Population Council

Follow this and additional works at: https://knowledgecommons.popcouncil.org/departments_sbsr-pgy

Part of the Demography, Population, and Ecology Commons, Family, Life Course, and Society Commons, Gender and Sexuality Commons, International Public Health Commons, and the Medicine and Health Commons How does access to this work benefit you? Let us know!

\section{Recommended Citation}

Amin, Sajeda and Althea D. Anderson. 2011. "Abordar la violencia sexual basada en el género (SGBV) contra niñas adolescentes," Promoción de transiciones a la vida adulta sanas, seguras y productivas Resumen no. 38. New York: Population Council. 


\section{Abordar la violencia sexual basada en el género (SGBV) contra niñas adolescentes}

\section{Elaborado por Sajeda Amin y Althea D. Anderson}

E I trabajo exhaustivo del Population Council en espacios seguros de adolescentes y programas de vida ha demostrado los caminos para otorgarle poder a las niñas adolescentes y los beneficios sustanciales para la sociedad (Levine et al. 2009; Mensch, Bruce, y Greene 1998; Chong, Hallman, y Brady 2006). El monitoreo e información de evaluación de estas iniciativas han producido evidencia empírica acerca de numerosas amenazas a la salud y bienestar de las niñas que se relacionan con las fuerzas sociales de larga data y las emergentes. Algunas prácticas de larga data como la exclusión de las mujeres de mercados laborales o el matrimonio prematuro de las niñas con hombres mayores, contribuyen a las inequidades del poder de género que están en la raíz de la violencia basada en el género. Mientras tanto, los cambios positivos en las condiciones sociales y económicas que han incrementado el logro educacional de las niñas y su participación en los mercados laborales formales también han imprevisto efectos negativos en la dinámica del poder de género. Estos incluyen incrementos en la participación de relaciones sexuales transaccionales de las niñas a cambio de cursos $y$ cuotas escolares, así como también vulnerabilidad ante la explotación sexual por empleadores en la fuerza de trabajo. En países desarrollados, donde el riesgo de resultados negativos de la salud sexual y reproductiva son altos y las niñas jóvenes están particularmente en alto riesgo, es importante abordar temas relacionados con la violencia sexual y basada en el género (SGBV) y las normas de género que se relacionen con estos riesgos.

La mayoría de los programas que abordan la violencia sexual basada en el género se focalizan en proporcionar servicios de salud clínica o servicios legales. Las intervenciones programáticas para la prevención de la violencia se fían de estudios correlativos para identificar los riesgos altos como la pobreza, vida urbana, educación pobre, enfermedades transmitidas sexualmente y factores de actitud como la aceptación de la comunidad de la violencia contra las mujeres. Estos enfoques programáticos buscan reducir la violencia ya sea con estrategias fijadas o con propuestas amplias para deslegitimar los comportamientos violentos. Estudios teóricos en SGBV enfatizan la pérdida de poder económico de las mujeres, la dependencia de las mujeres con los hombres y las estructuras sociales patriarcales que privilegian a los hombres de otra manera. Mientras que estos estudios nos ayudan a comprender los factores que eternizan la violencia, una teoría claramente articulada del

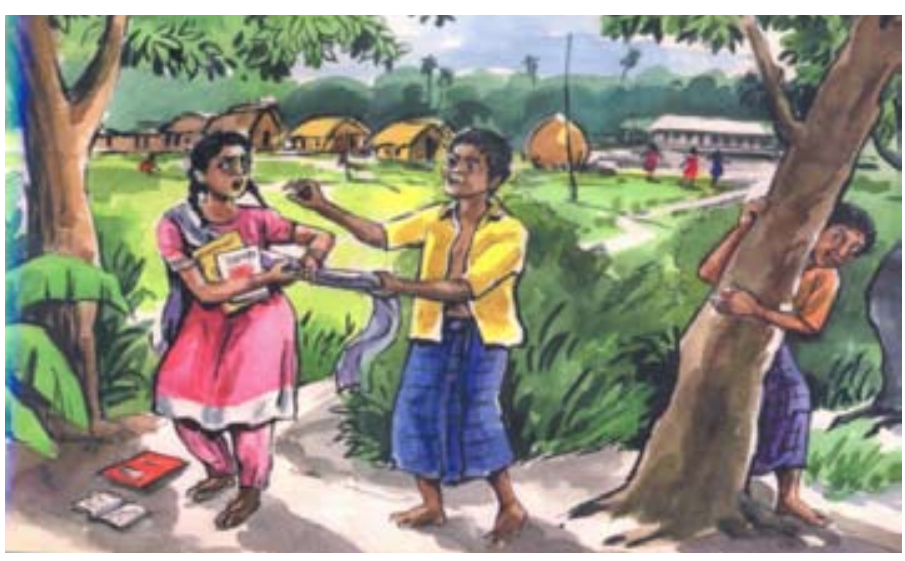

cambio de actitud y comportamiento, a menudo escasean en programas de prevención contra la violencia.

Las intervenciones para eliminar la SGBV incorporando enfoques basados en la comunidad, han sido colonizadas por Jewkes y colegas a lo largo del sur de África. Sus puntos de investigación para la importancia de las inequidades del poder de género, el estado dentro de grupos con compañeros masculinos y los patrones del derecho sexual masculino en construcciones sociales dominantes de masculinidad, actúan como determinantes de la SGBV (Wood and Jewkes 2001; Jewkes et al. 2009). La evidencia indica que las niñas se presentan más vulnerables debido a una combinación de género dominante y de jerarquías de edad que entorpece severamente la capacidad de las niñas para negarse ante los avances sexuales masculinos (Jewkes, Penn-Kekana, y Rose-Junius 2005). El trabajo del Population Council con adolescentes refuerza la importancia de puntos múltiples de acceso para cambiar las normas de la comunidad respecto a la SGBV. A pesar de que es esencial trabajar con niñas y mujeres para otorgarles poder y construir sus valores, no es suficiente si la comunidad no está comprometida.

\section{Focalizarse en niñas adolescentes}

El Council ha emprendido la investigación programática en las aptitudes e iniciativas de vida de las niñas adolescentes que pudieran además notificar un enfoque más integral para abordar la SGBV. Nuestro foco en las niñas jóvenes 
proviene del hecho de que la adolescencia es un momento crítico donde la vulnerabilidad de las mujeres se consolida. La experiencia del corte genital femenino, relaciones sexuales forzadas, infectarse con VIH, matrimonio de niños y maternidad durante la infancia, puede tener repercusiones inmediatas y duraderas. Las niñas que son las más vulnerables de la sociedad, incluyendo las que son pobres, huérfanas y están aisladas socialmente, generalmente están en alto riesgo. La evidencia del programa de niñas adolescentes del Council que busca reducir la vulnerabilidad durante este período crucial, es potencialmente útil al momento de crear intervenciones innovadoras para abordar la SGBV. De acuerdo a este objetivo, iniciamos las siguientes actividades:

1. Cosechar lecciones de nuestro trabajo sobre la mejora de la capacidad del sector de la salud para responder ante la violencia sexual;

2. Analizar las prácticas tradicionales nocivas como el matrimonio prematuro y el corte genital femenino para un mejor entendimiento contextual de la violencia basada en el género;

3. Extraer lecciones aprendidas de nuestros "espacios seguros" y del trabajo de exclusión social con niñas adolescentes para entender sus evaluaciones personales de vulnerabilidad y copiar estrategias con respecto a la SGBV;

4. Utilizar intervenciones programáticas con hombres y niños para cambiar las normas de género y promover formas más saludables de masculinidad como un enfoque principal para hacer que las comunidades y relaciones sean más seguras.

\section{Estrategias para abordar la SGBV}

La provisión de servicios de salud y legales para las víctimas de la violencia ha sido el foco de los esfuerzos programáticos para abordar la SGBV en muchas partes del mundo. Sin embargo, varios estudios muestran que los casos reportados de abuso sexual responden a solo una pequeña proporción de la verdadera incidencia de la SGBV (Heise, Ellsberg, y Gottmoeller 2002; Jewkes y Abrahams 2002; Koss, Heise, y Russo 1994; WHO 2005). Por otra parte, la mayoría de los programas de prevención que se ofrecen junto con programas de servicio alcanzan a mujeres que han sido víctimas de la SGBV. Mientras que puedan abordar formas de prevenir acontecimientos futuros para esos individuos, hay una necesidad de trabajar que aborda la prevención primaria cambiando las circunstancias que apoyan la SGBV.

Un hallazgo repetitivo de los informes del sector de la salud es que la mayoría de la gente que busca servicios luego de la violencia sexual son las niñas, a pesar de que muchos programas y protocolos dentro del sector de la salud están diseñados para adultos (Keesbury y Askew 2010). Las niñas jóvenes pueden estar cada vez más en riesgo de experimentar formas de violencia sexual sin denunciar, incluyendo relaciones sexuales no deseadas y forzadas y explotación sexual.

Un exclusivo enfoque judicial del sector de la salud se focalizó en la retribución y servicios para las víctimas, mientras que proporciona una solución para los resultados de la salud psíquica y mental de la SGBV pero no cuenta con medidas de prevención primaria. Hay una necesidad por identificar las causas de raíz de la violencia para diseñar puntos de intervención más apropiados. Un enfoque más exhaustivo para abordar la SGBV incluirá construir valores económicos y sociales para las niñas mientras que se compromete a los hombres y niños a promover normas de género más saludables y trabajar

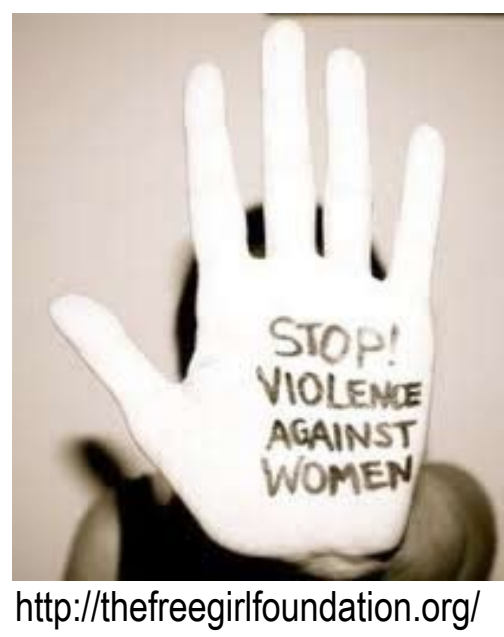

con comunidades para crear entornos más seguros. Tal enfoque cuenta con la ventaja agregada que puede ayudar a prevenir o retrasar el matrimonio de niños, el cual es cada vez más reconocido como forma de violencia basada en el género.

Para identificar un enfoque para la prevención de la SGBV, el Council ha revisado sus estrategias y resultados del programa de niñas adolescentes. El resumen muestra que la SGBV es una preocupación clave y un factor contextual importante que determina nuestro enfoque para trabajar con niñas. Hay una fuerte demanda de ejecutores y beneficiarios del programa por planes de estudio y estrategias para abordar la SGBV en programas de aptitudes de vida. Estos pueden incluir componentes pragmáticos que incorporan estrategias para reducir el aislamiento social y promover la inclusión de niñas y estrategias para comprometer a hombres y niños para hacer que la sociedad sea segura para las niñas.

Como respuesta a la conciencia creciente de los eslabones entre la SGBV, los derechos humanos y el desarrollo nacional, el Population Council, con el apoyo de Swedish-Norwegian Regional VIH and AIDS Team for Africa, inició una red de SGBV de países múltiples en 2006. Esta red incluye a ocho agencias asociadas en el Este y Sur de África que están implementando proyectos que abordan los determinantes y las consecuencias de la SGBV de forma integrada. Estas agencias refuerzan los programas nacionales para encontrar mejor las necesidades médicas, psicológicas y judiciales de las sobrevivientes a la SGBV, mientras que mejoran las estrategias para su prevención (Population Council 2008).

\section{Construir valores sociales}

Nuestras intervenciones de "espacios seguros" ofrecen varios caminos para abordar la SGBV a través de estrategias de prevención primaria. Los espacios seguros otorgan una oportunidad para que las niñas construyan valores sociales y humanos y aumenten el acceso a las redes sociales en espacios físicamente seguros. Dentro de estos espacios, las niñas pueden promover las conexiones sociales que contribuyen a la reducción de riesgos a través de la afiliación e identidad grupal, lo cual mejora las habilidades de negociación y comunicación y genera confianza en sí mismas. (Austrian 2011; Austrian y Ghati 2010).

La inversión del Council para crear acceso a espacios seguros está manejada por nuestro trabajo en abordar la exclusión social de las niñas

2 - Visite www.popcouncil.org/publications/serialsbriefs/TABriefs.asp para obtener todos los resúmenes de la serie Promoting healthy, safe, and productive transitions to adulthood (Promoción de transiciones a la vida adulta sanas, seguras y productivas). 
adolescentes. En Sudamérica, por ejemplo, las investigaciones del Council analizaron los efectos de la inseguridad personal en la vida diaria de hombres y mujeres jóvenes. El Council y Crime Reduction in Schools Project (CRISP) (Proyecto de reducción de crímenes en escuelas) condujeron talleres grupales con juventud que vivía fuera de Durban, Sudáfrica, para planear espacios importantes en sus comunidades y luego estimar estos espacios en términos de su seguridad. Los hallazgos indican que las niñas identificaron más peligros físicos que los niños, muchos de los mismos asociados al riesgo de abuso y violación sexual. Además, las niñas jóvenes (de entre 10 y 13 años) fueron las más vulnerables y no pudieron identificar un sólo espacio en sus comunidades al que reconocieran más que "algo seguro" (Kenworthy, Hallman, y Diers 2008). Nuestra investigación sobre la exclusión social en Sudáfrica demuestra que las niñas adolescentes tienen un acceso restringido a las redes sociales. En general, la adolescencia es un momento en el que los jóvenes adultos expanden sus horizontes geográficos e intelectuales; sin embargo, para las niñas vulnerables las conexiones y el acceso social a los recursos de la comunidad reducen considerablemente durante este período. Nuestros hallazgos de Sudáfrica muestran que el aislamiento social está asociado con el riesgo de las niñas de experimentar violencia sexual (ver Figura 1).

En el vecindario de Kibera de Nairobi, Kenya, la investigación del Council ha destacado que las experiencias de violencia de adolescentes ya se relacionan a una escasez de espacios seguros. En un estudio en el 2006 basado en la población de adolescentes en el barrio de Kibera, la gente joven indicaba que habían sido testigos y experimentado violencia considerable y amenazas para la seguridad, con muchas - especialmente niñas - que tenían redes de apoyo o de seguridad limitadas. Las niñas adolescentes en particular, experimentan violencia, acoso, coacción y violación. El cuarenta y tres por ciento de las niñas ha experimentado sus primeras relaciones sexuales forzadas. Además, las niñas adolescentes casadas informaron altos índices de violencia doméstica (Erulkar y Matheka 2007). Una intervención que busca abordar la violencia en el centro Binti Pamoja, un programa para niñas adolescentes de entre 10 y 22 años en Kibera. Binti Pamoja le otorga a las niñas espacios seguros y mentores a través de los cuales pueden aprender salud sexual y reproductiva, desarrollo adolescente y educación financiera y desarrollar amistades y habilidades en la comunicación, negociación y liderazgo. Estos programas ofrecen actividades críticas que abordan las preocupaciones de salud y proporcionan otorgamiento de poder social y económico para las niñas. El Council se asoció con Binti Pamoja y otras ONG locales para diseñar programas construidos en las habilidades y para monitorear y evaluar su efecto en fortalecer los valores económicos y sociales y oportunidades para niñas en Kibera (Austrian 2011).

\section{Asegurar valores económicos}

Las experiencias de niñas adolescentes de la SGBV también han informado estrategias programáticas para inculcar alfabetización financiera y aptitudes de vida brindadas en un contexto de espacios seguros. En Bangladesh, el Council colaboró con el Institute of Development Studies (Instituto de Estudios de Desarrollo) de Bangladesh para evaluar a Kishori Abhijan (Aventura de niñas adolescentes), un programa de vida modelado en el marco de espacios seguros. El programa aumentó significativamente las oportunidades laborales y el conocimiento por la salud de las niñas. Para niñas más jóvenes (de entre
Figura 1. Las niñas con menos conexiones sociales son más propensas a experimentar relaciones sexuales forzadas (Siempre forzadas físicamente: niñas de entre 14 y 16 años)

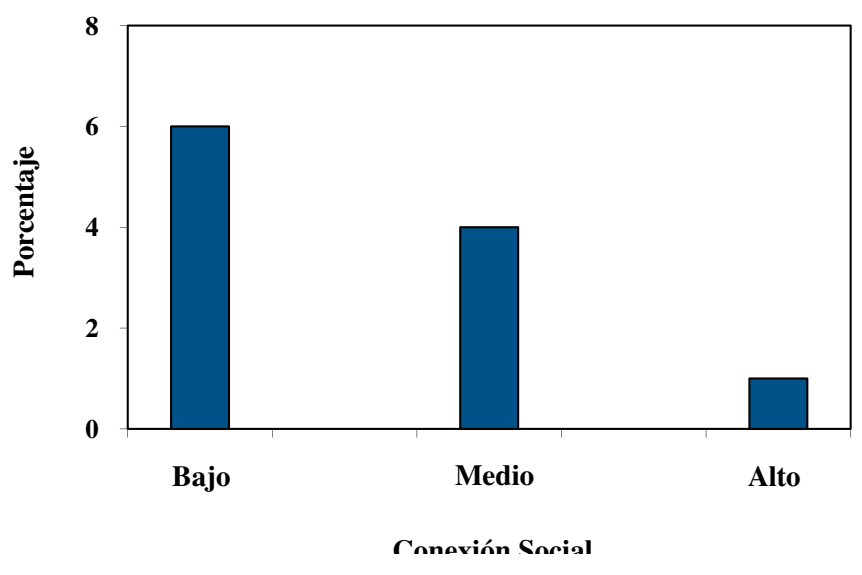

Fuente: Hallman y Roca 2011

12 y 14 años) en el distrito más pobre, el programa contribuyó para mejorar el índice de inscripción en escuelas y disminuir el índice de matrimonio (Amin y Suran 2005). Los investigadores también descubrieron que los participantes de Kishori Abhijan habían formado un sentido más fuerte de autoestima y conexión comparado con las niñas que no estuvieron expuestas a la intervención. Las niñas en el programa estuvieron más dispuestas a informar el haber tenido amigos en el mismo pueblo y tuvieron movilidad social mayor (Amin 2007). Los investigadores del Council también implementaron la educación financiera y programas de ahorro en Kenia y Uganda y los programas de alfabetización financiera en Sudáfrica (Austrian 2011; Hallman 2011).

\section{Direcciones futuras}

Trabajar con hombres y niños

Mientras que las iniciativas para las niñas son críticas, su otorgamiento de poder y avance a la larga dependen de la transformación de las normas de la comunidad. El hecho de crear comunidades más seguras requiere del compromiso de hombres y niños y de miembros de la comunidad para complementar los programas de las niñas. El Population Council y sus socios han trabajado con niños y hombres en Brasil, Etiopía e India en temas de masculinidad y desigualdad de género y han demostrado el impacto de programas de comportamiento que reducen la violencia auto reportada cometida por hombres y niños (Erulkar y Alemayehu 2009; Pulerwitz y Barker 2008; Verma et al. 2006). El proyecto Addis Birhan del Council en la región Amhara de Etiopía compromete a hombres en conversaciones comunitarias acerca de las normas de género disfuncionales y su cuota y costos para mujeres y niños. Este programa ha generado cambios positivos en los comportamientos y actitudes de los hombres hacia la masculinidad, como fue informado por hombres y sus jóvenes esposas (Erulkar y Alemayehu 2009). Planeamos construir sobre estas iniciativas con hombres y niños para ampliar el concepto de espacios seguros para comunidades y relaciones seguras. 


\section{Crear comunidades seguras}

Nuestra experiencia al trabajar con hombres y niños ha demostrado resultados prometedores y se ajusta con varios estudios que comenzaron a demostrar la viabilidad y efectividad de cambiar las normas de género y reducir la SGBV a través de intervenciones a nivel comunitario (Barker et al. 2010). E enfoque central de estos programas es comprometer a hombres y niños en conversaciones acerca de su masculinidad, analizar los problemas asociados y proponer un cambio aceptable. La clave es aplicar un modelo positivo más que punitivo del cambio de comportamiento con hombres y niños, identificar la forma en la que dicho cambio es en su propio beneficio. El Council seguirá focalizándose en programas de cambio de comportamiento para hombres y niños que demuestren normas de género positivas, mientras se crea un entorno de apoyo para adoptar estas normas. De forma similar, las estrategias para prevenir la violencia a nivel comunitario podrían hacer avanzar los programas de cambio de comportamiento para las niñas que promueven la aceptación de comportamientos seguros y firmes por niñas, mientras se les otorga poder para adoptar estos cambios.

\section{Referencias y fuentes}

Amin, Sajeda. 2007. "Empowering adolescent girls in rural Bangladesh: Kishori Abhijan." Nueva York: Population Council.

Amin, Sajeda y Luciana Suran. 2005. "Program efforts to delay marriage through improved opportunities: some evidence from rural Bangladesh," Paper presented at XXV International Population Conference of the International Union for the Scientific Study of Population conference, Tours, Francia, 18-23 de julio. <http://iussp2005. princeton.edu/download.aspx?submissionld=52255>.

Austrian, Karen. 2011. "Expanding Safe Spaces, Financial Education, and Savings for Adolescent Girls in Kenya." Promoting Healthy, Safe, and Productive Transitions to Adulthood Brief no. 29. Nueva York, NY: Population Council.

Austrian, Karen y Dennitah Ghati. 2010. Girl Centered Program Design: A Toolkit to Develop, Strengthen and Expand Adolescent Girls Programs. Population Council.

Barker, Gary, Christine Ricardo, Marcos Nascimento, et al. 2010. "Questioning gender norms with men to improve health outcomes: Evidence of impact." Global Public Health 5(5): 539-553

Chong Erica, Kelly Hallman y Martha Brady. 2006. "Investing when it counts: Generating the evidence base for policies and programmes for very young adolescents-Guide and tool kit." Nueva York, NY: Population Council.

Erulkar, Annabel S. y Awraris Alemayehu. 2009. "Addis Birhan ('New Light'): Fostering husbands' involvement and support in Amhara Region, Ethiopia," Ethiopia Program Brief Series. Etiopía: Population Council.

Erulkar, Annabel S. y James K. Matheka. 2007. "Adolescence in the Kibera slums of Nairobi." Nairobi, Kenia: Population Council.

Hallman, Kelly, Kasthuri Govender, Eva Roca, Rob Pattman, Emmanual Mbatha y Deevia Bhana. 2011. "Enhancing financial literacy, VIH/AIDS skills, and safe social spaces among vulnerable South African youth." Promoting Healthy, Safe, and Productive Transitions to Adulthood Brief no. 4. Nueva York: Population Council.
Hallman, Kelly y Eva Roca. 2011. "Reducing the social exclusion of girls," Promoting Healthy, Safe, and Productive Transitions to Adulthood Brief no. 27. Nueva York, NY: Population Council.

Heise, Lori, Mary C. Ellsberg y M. Gottmoeller. 2002. "A global overview of genderbased violence." International Journal of Gynecology and Obstetrics 78(Suppl. 1): S5-S14.

Kenworthy, Nora, Kelly Hallman y Judith A. Diers. 2008. "Identifying sources of adolescent exclusion due to violence: Participatory mapping in South Africa." Promoting Healthy, Safe, and Productive Transitions to Adulthood Brief no. 30. Nueva York, NY: Population Council.

Jewkes, Rachel, Yandisa Sikweyiya, Robert Morrell y Kristen Dunkle. 2009. "Understanding Menís health and Use of Violence: Interface of Rape and VIH in South Africa," Gender and Health Research Unit Report. Pretoria: South African Medical Research Council.

Jewkes, Rachel, Loveday Penn-Kekana, y Hetty Rose-Junius. 2005. "'If they rape me, I canit blame them': Reflections on gender in the social context of child rape in South Africa and Namibia." Social Science \& Medicine 61:1809-1820.

Jewkes, Rachel y Naeema Abrahams. 2002. "The epidemiology of rape and sexual coercion in South Africa: an overview." Social Science \& Medicine. 55(7): 1231 1244.

Keesbury, Jill e lan Askew. 2010. "Comprehensive responses to gender-based violence in low-resource settings: Lessons learned from implementation." Nueva York, NY: Population Council.

Koss, Mary P., Lori Heise y Nancy F.Russo. 1994. "The global health burden of rape." Psychology of Women Quarterly 18: 509-537.

Levine, Ruth, Cynthia B. Lloyd, Margaret Greene y Caren Grown. 2009. "Girls Count: A Global Investment \& Action Agenda." Washington, DC: Center for Global Development.

Mensch, Barbara, Judith Bruce y Margaret Greene. 1998. The Uncharted Passage: Girls'Adolescence in the Developing World. Nueva York, NY: Population Council.

Population Council. 2008. "Africa regional sexual and gender-based violence network consultation: Technical exchange and planning meeting." Nueva York, NY: Population Council.

Pulerwitz, Julie y Gary Barker. 2008. "Measuring attitudes toward gender norms among young men in Brazil: Development and psychometric evaluation of the GEM scale." Men and Masculinities 10(3): 322-338.

Verma, Ravi K., Julie Pulerwitz, Vaishali Mahendra, Sujata Khandekar, Gary Barker, P. Fulpagare, and S.K. Singh. 2006. "Challenging and changing gender attitudes among young men in Mumbai, India." Reproductive Health Matters 14(28): 135-143.

Wood, Katherine y Rachel Jewkes. 2001. "'Dangerous' love: Reflections on violence among Xhosa township youth," in R. Morrell (ed.), Changing Men in Southern Africa. Pietermaritzburg: University of Natal Press.

World Health Organization. 2005. "WHO multi-country study on women's health and domestic violence against women: Summary report of initial results on prevalence, health outcomes and women's responses." Ginebra.

\section{Population Council}

El Population Council cambia la manera en la que el mundo piensa acerca de los problemas de salud y desarrollo. Buscamos entender las causas y las consecuencias de la desigualdad de género y las disparidades en las oportunidades que surgen durante la adolescencia. Brindamos las evidencias para desarrollar mejores programas y políticas prácticas que garanticen una transición exitosa y productiva hacia la edad adulta en los países en vías de desarrollo. www.popcouncil.org

() 2011 The Population Council, Inc.

4 - Visite www.popcouncil.org/publications/serialsbriefs/TABriefs.asp para obtener todos los resúmenes de la serie Promoting healthy, safe, and productive transitions to adulthood (Promoción de transiciones a la vida adulta sanas, seguras y productivas). 